\title{
Aplastic Anemia and Good Syndrome in a Heavily Treated Stage IV Thymoma Patient: A Case Report and Review of the Literature
}

\author{
Sofia Chiatamone Ranieri (D), ${ }^{1}$ Stefania Trasarti (D, ${ }^{1}$ Maria Antonietta Arleo, ${ }^{1}$ \\ Luisa Bizzoni, ${ }^{1}$ Livia Bonanni, ${ }^{2}$ Valeria Di Battista, ${ }^{3}$ Maria Assunta Limongiello, ${ }^{1}$ \\ Maria Grazia Nardacci, ${ }^{1}$ Giuseppe Gentile, ${ }^{1}$ and Robin Foà ${ }^{1}$ \\ ${ }^{1}$ Department of Translational and Precision Medicine, University of Rome "Sapienza", Rome, Italy \\ ${ }^{2}$ Department of Molecular Medicine, University of Rome "Sapienza", Rome, Italy \\ ${ }^{3}$ Department of Medicine, Center of Hemato-Oncology Research (C.R.E.O.), University of Perugia, Perugia, Italy \\ Correspondence should be addressed to Stefania Trasarti; trasarti@bce.uniroma1.it
}

Received 30 June 2019; Accepted 24 October 2019; Published 12 November 2019

Academic Editor: Sergio Storti

Copyright (C) 2019 Sofia Chiatamone Ranieri et al. This is an open access article distributed under the Creative Commons Attribution License, which permits unrestricted use, distribution, and reproduction in any medium, provided the original work is properly cited.

\begin{abstract}
Thymoma is an uncommon slowly growing neoplasm. It usually presents with paraneoplastic syndromes including the immunodeficiency syndrome called Good syndrome and hematological disorders. Pure red cell aplasia is a well-recognized complication of thymoma, and aplastic anemia is very rare in association with GS. We report a case of GS in a heavily treated patient with stage IV thymoma associated with a pure red cell aplasia and an amegakaryocytic thrombocytopenia that evolved into an AA and provide an up-to-date review of the relevant literature. This is the first case of the association of GS and AA with the coexistence of a heavily treated stage IV thymoma. The fatal outcome was not related to the progression of the thymoma, but rather to the severe infectious complications. The combination of lymphopenia and hypogammaglobulinemia typical of GS, coupled to the neutropenia, caused by bone marrow failure, was the main predisposing factor for the unfavourable outcome.
\end{abstract}

\section{Case Presentation}

In October 2015, a 53-year-old man was admitted to our hospital due to an incidental finding of severe anemia. The patient had a long medical history of thymoma (B2/B3) started in 1981 with pleural and lymphatic metastatic repetitions and Masaoka stage IVb and received chemo-, immuno-, and radiotherapy following multiple recurrences. He underwent five surgical excisions and five traditional lines of chemotherapy associated with radiotherapy to the mediastinum and paravertebral tissue. Due to the progression of the disease, he was enrolled in different experimental protocols including receptor radionuclide therapy with somatostatin analogue, sorafenib, volasertib, nintedanib, and everolimus. From October 2013, he started a maintenance treatment with low-dose cyclophosphamide plus octeotride. Periodic CT/PET scans showed a stable disease with paracostal, paracardiac, paravertebral, and paramediastinic residual metastatic nodules.

When the patient was admitted to our institute (October 2015), the complete blood count was as follows: red blood cells $2.08 \times 10^{12} / \mathrm{L}$, hemoglobin $6.9 \mathrm{~g} / \mathrm{dL}$, mean corpuscular volume $95 \mathrm{fL}$, mean hemoglobin concentration $34.1 \mathrm{~g} / \mathrm{dL}$, white blood cells $6.7 \times 10^{9} / \mathrm{L}$ (neutrophils $51 \%$ and lymphocytes $7 \%$ ), platelets $447 \times 10^{9} / \mathrm{L}$, and reticulocyte count $0.2 \%$. Serologies and DNA tests were negative for viral infections (CMV, EBV, HIV, and Parvovirus). No immunological abnormalities were detected, except for positive antiacetylcholine receptor antibodies and reduced levels of immunoglobulin isotypes IgG, IgA, and IgM. A bone marrow aspirate and biopsy revealed a virtually absent erythropoiesis with a preserved granulocytic and megakaryocytic maturation without infiltration by thymocytes and CD1a + lymphocytes (Figure 1-2015). A myelodysplastic 
2015

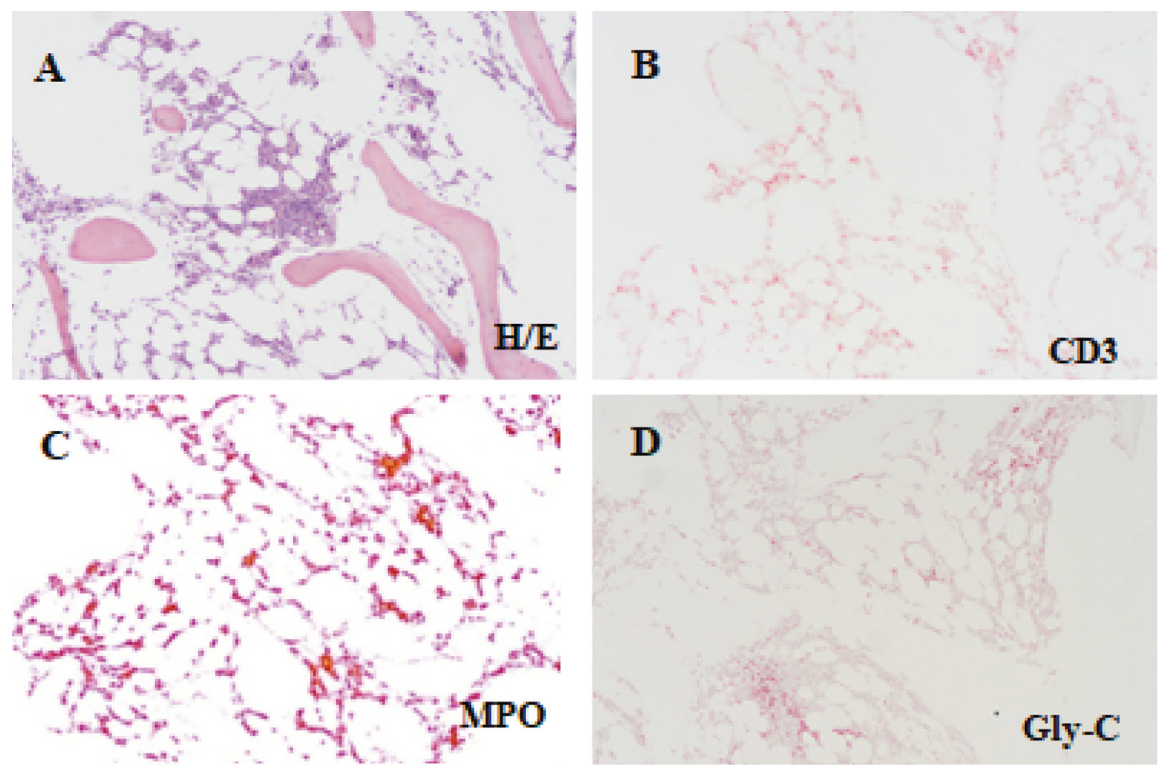

2017
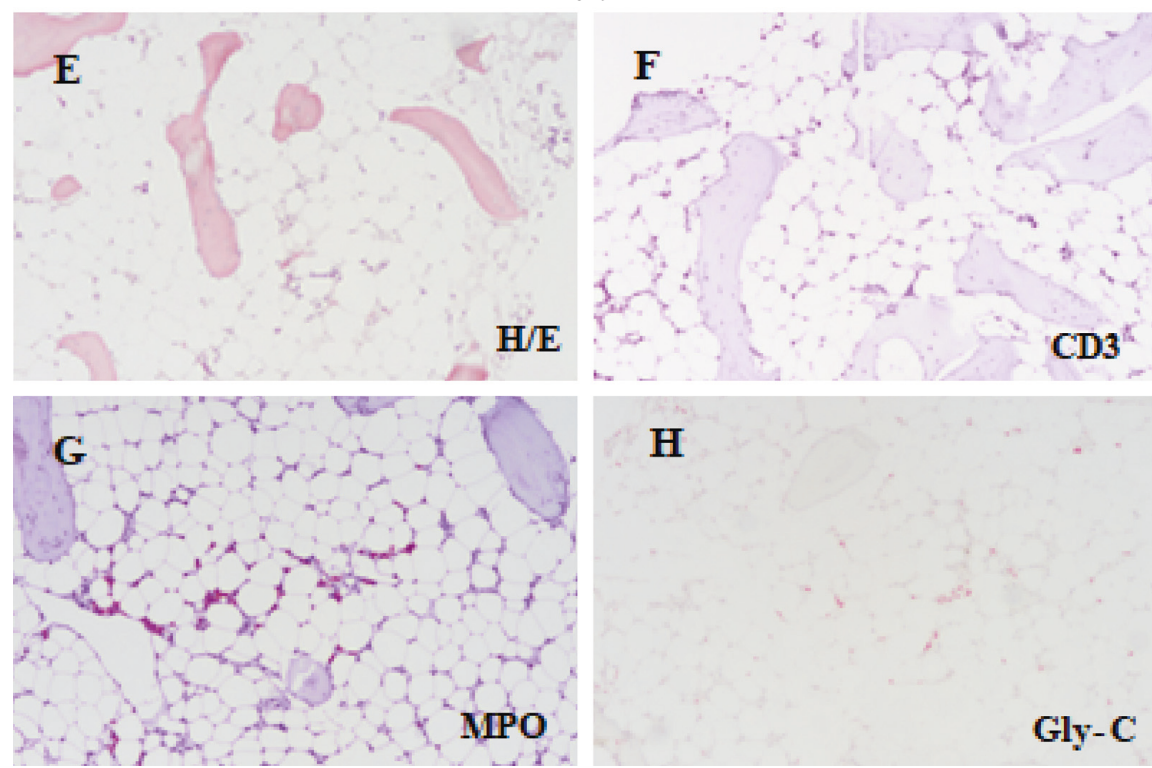

$\mathbf{H}$

\section{Gly-C}

Figure 1: Histopathological images of the bone marrow (magnification 10x). (a) Biopsy section obtained on October 2015 showing a hypocellular bone marrow with preserved megakaryopoiesis (hematoxylin and eosin stain). (c) Myeloperoxidase staining revealed adequate numbers of myeloid cells. (d) Glycophorin C staining revealed a virtually absent erythropoiesis. (e) Biopsy section obtained on March 2017 revealed a severe hypocellular marrow ( $<5 \%$ ) (hematoxylin and eosin stain). (g) Myeloperoxidase staining revealed a virtually absent myelopoiesis. (h) Glycophorin C staining revealed a virtually absent erythropoiesis. (b and f) In both biopsies, immunohistochemical stain for $\mathrm{CD} 3$ revealed a modest lymphoid component with interstitial distribution (CD3+).

syndrome was excluded by morphological evaluation, cytogenetic analysis, and mutational analysis, which revealed a normal karyotype and the absence of EZH2, GATA2, and TET2 mutations [1]. We also searched for rare mutations associated to sideroblastic anemia such as TRNT1. On this basis, we excluded the diagnosis of congenital sideroblastic anemia.

The study of the peripheral blood lymphocyte subpopulations showed the absence of B lymphocytes and an inversion of the CD4/CD8 ratio within the sCD3+ $\mathrm{T}$ lymphocytes, without a clonal restriction. Based on these findings, a diagnosis of PRCA associated to GS was made [2]. Cyclophosphamide was discontinued, and the patient remained on octeotride. The patient started supportive therapy with weekly blood transfusions, subcutaneous gammaglobulin (IG) infusions every three weeks, and erythropoietin and prednisone $75 \mathrm{mg}$ daily. The last two drugs were suspended after 2 months due to lack of response. In May 2016, the patient developed a severe thrombocytopenia (platelets $15 \times 10^{9} / \mathrm{L}$ ). A bone marrow aspirate revealed a new marked reduction in megakaryocytes, and data were compatible with a diagnosis of AATP 
[3]. Treatment with high-dose ivIG (1gr/Kg) and steroids was started, which was followed by a slow and partial response that persisted for 9 months. Unfortunately, in February 2017, the patient showed again a severe thrombocytopenia (platelets $20 \times 10^{9} / \mathrm{L}$ ), unresponsive to steroids and high-dose ivIG. After Institutional Ethical Committee approval, eltrombopag was started as compassionate therapy at $50 \mathrm{mg}$ daily dose and thereafter increased to $100 \mathrm{mg}$ daily. After 4 weeks, the patient presented a marked neutropenia, and a bone marrow biopsy showed a final evolution to AA (Figure 1-2017).

Treatment with growth factors was initiated without benefit. The patient was not considered eligible to transplant because of the persistent thymoma and severe immunodepression due to GS and previous multiple chemotherapic treatment, so antithymocyte globulin was proposed [4]. After the prephase treatment with high-dose steroids, the patient developed a digestive bleeding associated to an invasive aspergillosis with fever and a severe hemorrhagic cystitis. The patient finally died of infectious complications.

\section{Discussion and Conclusions}

To our knowledge, this is the first case of the association of GS and AA with the coexistence of a heavily treated stage IV thymoma. GS is a rare association of thymoma and immunodeficiency first described more than 50 years ago. It is defined by low or absent B-cells in the peripheral blood, hypogammaglobulinemia and (in some cases) defects in cellmediated immunity (CD4 $+\mathrm{T}$ lymphopenia and an inverted $\mathrm{CD} 4+/ \mathrm{CD} 8+\mathrm{T}$-cell ratio), and susceptibility to infections [2]. PRCA, thought to be due to T-cell-mediated destruction of erythroid progenitor cells, is reported in $2 \%$ of patients with thymoma and is characterized by anemia, reticulocytopenia, and severe bone marrow erythroid hypoplasia [2]. AATP is a rare immune-mediated disorder characterized by thrombocytopenia resulting from an unexplained reduction in the number of bone marrow megakaryocytes in the presence of an otherwise normal hematopoiesis. The exact prevalence of amegakaryocytic thrombocytopenia is unknown. The available literature comprises case reports and small case series [5]. AA is an uncommon complication of thymic tumors $(0-1.4 \%)$. It is characterized by bone marrow failure. Treatment of PRCA, AATP, and AA is based on immunosuppressive drugs; there is, however, no consensus on the optimal therapeutic strategy $[2,4,5]$. The association of GS, PRCA, and AATP evolving into an AA is extremely rare. There is a series of cases reported with a concomitant PRCA and GS [6], cases of thymoma with AA [7], and 7 cases of PRCA and AATP [3, 8-13], 3 of which progressed to AA $[3,8,9]$, but never with evidence of a GS.

Very few cases of AA in GS have been described in the literature with many of them being associated with benign spindle cell tumors [14-16], different from our case (Table 1). In 3/6 cases, the diagnosis of GS and AA was simultaneous with the diagnosis of thymoma $[14,15,18]$, and in 1 case, thymectomy was performed but without remission of the AA [14]. In 2 cases, the diagnosis of GS associated with a PRCA which then evolved into an AA was made years after the onset of the thymoma $[16,17]$; in one of the 2 cases, surgery and local chemotherapy instillations were carried out after the onset of anemia, but they were ineffective for aplasia although there was no recurrence of thymoma [17]; and in the other case, there are insufficient data to define the state of the illness [16]. Five of the 6 cases died of infectious and hemorrhagic or thrombotic complications. In the more recently described case of pancytopenia, this appeared three years after thymectomy and without evidence of cancer recurrence, and the patient responded to eltrombopag [19].

In a recent review of $\mathrm{AA}$ and thymoma, cyclosporine therapy, both either alone or in combination with other agents such as ATG and G-CSF, was associated with a response rate of $66.6 \%$ [7]. Furthermore, there are 4 reports which have described patients who underwent an allogenic hematopoietic stem cell transplantation for aplastic anemia, 2 of which with metastatic thymoma, nobody with GS. All showed a good response [8, 20-22]. Considering the refractoriness of the tumor, in our case the administration of immunosuppressive agents to prevent tumor progression was excluded [23]; unfortunately, the response to corticosteroids and high-dose iv IG was ineffective for the PRCA and partially effective for the AATP and failed to contain the progression to AA.

ATG was proposed, but the patient died after the prephase treatment due to invasive aspergillosis. In view of the promising results obtained in AA [24] and in a case of GS with pancytopenia [19], we attempted to use eltrombopag, but could not assess its effectiveness due to the short time of administration. The prognosis of GS is considered worse than that of other primary immunodeficiencies, with an overall mortality rate of $46 \%$ [2].

The combination of lymphopenia and hypogammaglobulinemia typical of GS, coupled to the neutropenia, caused by bone marrow failure, was the main predisposing factor for the poor outcome of our patient [25].

In the literature, there have been 30 cases of AATP without significant comorbidities and autoimmune disease that have also shown a rapid progression to AA [26]. The rapid progression of AATP to AA in these cases suggests that thymoma-associated AATP may have a more aggressive disease course than thymoma-associated PRCA. In our case, the previous and prolonged chemo-, radio-, and immunotreatment could have caused a severe immunodepression with an acquired immunodeficiency syndrome (as observed, for example, in patients transplanted or subjected to long-term immunosuppressive treatments) associated with clonal/dysplastic changes responsible for the aplasia as observed in our patient. For this reason, our first goal was to exclude a myelodysplastic syndrome secondary to chemotherapy and radiotherapy treatments: no morphological changes suggestive of MDS were observed, the karyotype was normal, and also the mutations associated with myelodysplasia and rare forms of sideroblastic anemia associated with immunodeficiency such as TET2, GATA2, TRNT1, were negative.

On the other hand, the presence in the bone marrow of an expansion of $\mathrm{T}$ lymphocytes in the absence of clonality, the inversion of the CD4/CD8 ratio observed at the time of immunophenotyping, and the selective absence of B lymphocytes associated with the presence of selective red 


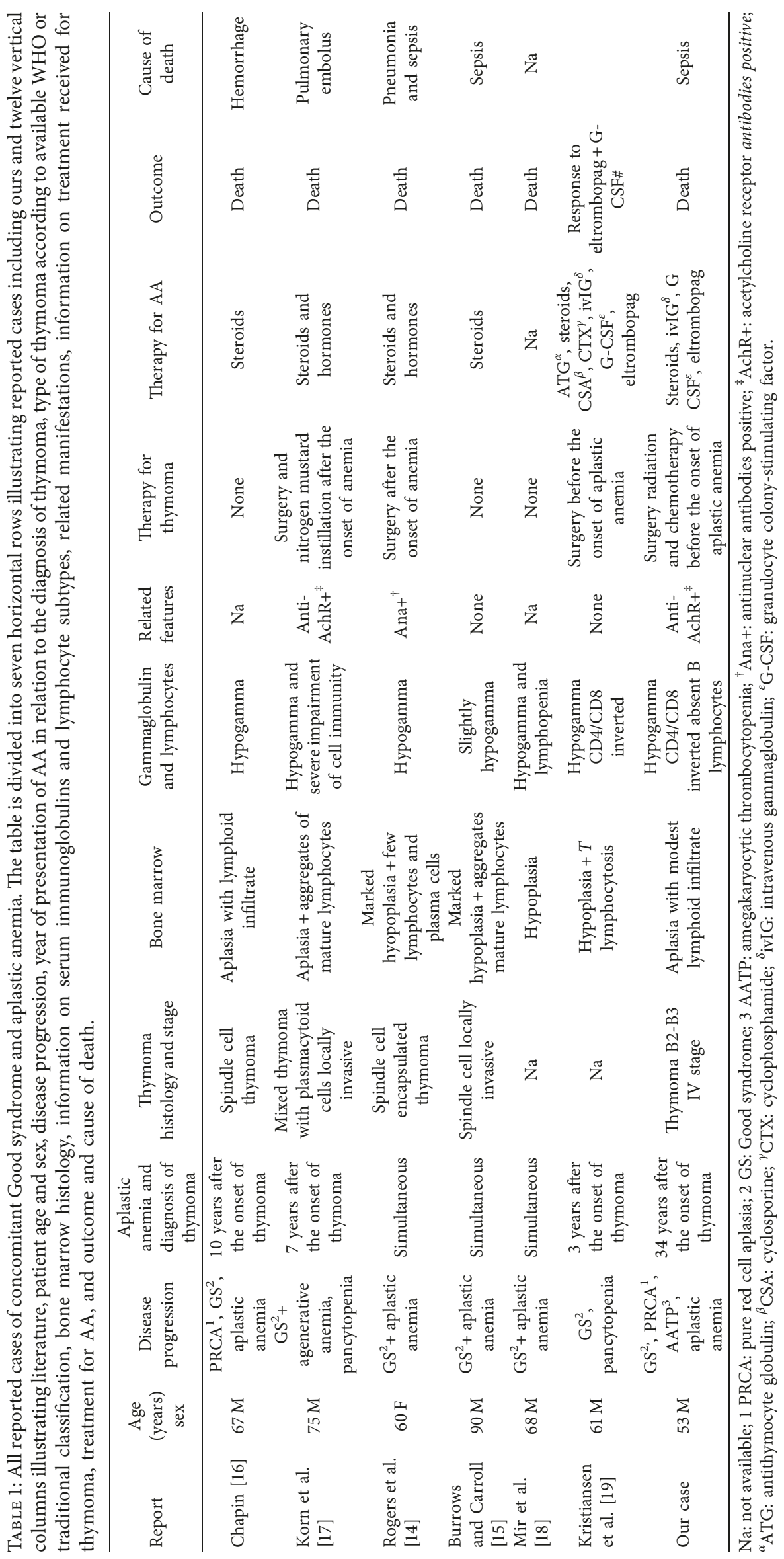


series aplasia led us to believe that it was a paraneoplastic picture of altered maturation and disreactivity of autoimmune T lymphocytes. Before our clinical evaluation, we had no evidence of an immunoglobulin dosage, so we could not date the onset of Good syndrome. We only know that the patient has never had serious infections during previous treatments.

Every time a recurrence of the thymic disease occurred in our patient, therapy was immediately started (chemotherapy, radiotherapy, or immunotherapy) and complete remission was readily observed. Instead, at the time of our evaluation, the patient presented with a persistent although stable thymicmetastatic disease for at least 2 years, in maintenance with octeotride and cyclophosphamide. Therefore, it is assumed that the persistence of the thymic disease resulted in secondary pathologies such as PRCA, than evolved in AA and GS.

Our case highlights the challenging issue of treating a young fit patient with GS and AA, for whom, to date, we have no effective therapeutic tools. We describe this case not only for its rarity, but also for the complexity of the clinical management in an attempt to improve our understanding of this difficult-to-treat entity.

\section{Abbreviations}

$\begin{array}{ll}\text { Na: } & \text { Not available } \\ { }^{\dagger} \text { Ana+: } & \text { Antinuclear antibodies positive } \\ { }^{\ddagger} \text { AchR+: } & \text { Acetylcholine receptor antibodies positive } \\ { }^{\alpha} \text { ATG: } & \text { Antithymocyte globulin } \\ { }^{\beta} \text { CSA: } & \text { Cyclosporine } \\ { }^{\gamma} \text { CTX: } & \text { Cyclophosphamide } \\ \delta_{\text {ivIG: }} & \text { Intravenous gammaglobulin } \\ { }^{\varepsilon} \text { G-CSF: } & \text { Granulocyte colony-stimulating factor } \\ \text { AA: } & \text { Aplastic anemia } \\ \text { GS: } & \text { Good syndrome } \\ \text { AATP: } & \text { Amegakaryocytic thrombocytopenia } \\ \text { PRCA: } & \text { Pure red cell aplasia } \\ \text { CT/ } & \text { Computed tomography/positron emission } \\ \text { PET: } & \text { tomography } \\ \text { CMV: } & \text { Cytomegalovirus } \\ \text { EBV: } & \text { Epstein-barr virus } \\ \text { HIV: } & \text { Human Immunodeficiency virus } \\ \text { ATG: } & \text { Antithymocyte globulin. }\end{array}$

\section{Consent}

Written informed consent was obtained from the patient's next-of-kin for publication of this case report and any accompanying images. A copy of the written consent is available for review by the editor-in-chief of this journal.

\section{Conflicts of Interest}

The authors declare that they have no conflicts of interest.

\section{Authors' Contributions}

Stefania Trasarti conceptualized the study. Stefania Trasarti and Sofia Chiatamone Ranieri designed the study. Stefania Trasarti, Sofia Chiatamone Ranieri, Maria Antonietta Arleo,
Maria Assunta Limongiello, Maria Grazia Nardacci, and Valeria Di Battista acquired the data. Stefania Trasarti, Sofia Chiatamone Ranieri, and Giuseppe Gentile analyzed and interpreted the data. Stefania Trasarti and Sofia Chiatamone Ranieri prepared the manuscript. Stefania Trasarti and Giuseppe Gentile edited the manuscript. Giuseppe Gentile and Robin Foà reviewed the manuscript.

\section{References}

[1] M. Haider, E. J. Duncavage, K. F. Afaneh, R. Bejar, and A. F. List, "New insight into the biology, risk stratification, and targeted treatment of myelodysplastic syndromes," American Society of Clinical Oncology Educational Book, vol. 37, pp. 480-494, 2017.

[2] C. Bernard, H. Frih, F. Pasquet et al., "Thymoma associated with autoimmune diseases: 85 cases and literature review," Autoimmunity Reviews, vol. 15, no. 1, pp. 82-92, 2016.

[3] I. Maslovsky, D. Gefel, L. Uriev, D. Ben Dor, and G. Lugassy, "Malignant thymoma complicated by amegakaryocytic thrombocytopenic purpura," European Journal of Internal Medicine, vol. 16, no. 7, pp. 523-524, 2005.

[4] E. Di Bona, F. Rodeghiero, B. Bruno et al., "Rabbit antithymocyte globulin (r-ATG) plus cyclosporine and granulocyte colony stimulating factor is an effective treatment for aplastic anaemia patients unresponsive to a first course of intensive immunosuppressive therapy," British Journal of Haematology, vol. 107, no. 2, pp. 330-334, 1999.

[5] N. Agarwal, J. E. Spahr, T. L. Werner, D. L. Newton, and G. M. Rodgers, "Acquired amegakaryocytic thrombocytopenic purpura," American Journal of Hematology, vol. 81, no. 2, pp. 132-135, 2006.

[6] T. Kelesidis and O. Yang, "Good's syndrome remains a mystery after 55 years: a systematic review of the scientific evidence," Clinical Immunology, vol. 135, no. 3, pp. 347-363, 2010.

[7] A. V. Chintakuntlawar, S. A. Rizvi, S. D. Cassivi, and A. Pardanani, "Thymoma-associated pancytopenia: immunosuppressive therapy is the cornerstone for durable hematological remission," Annals of Hematology, vol. 94, no. 3, pp. 453-458, 2015.

[8] A. Simkins, A. Maiti, N. J. Short et al., "Acquired amegakaryocytic thrombocytopenia and red cell aplasia in a patient with thymoma progressing to aplastic anemia successfully treated with allogenic stem cell transplantation," Hematology/ Oncology and Stem Cell Therapy, vol. 12, no. 2, pp. 115-118, 2019.

[9] S. Dahal, E. Sharma, S. Dahal, B. Shrestha, and B. Bhattarai, "Acquired amegakaryocytic thrombocytopenia and pure red cell aplasia in thymoma," Case Reports in Hematology, vol. 2018, Article ID 5034741, 3 pages, 2018.

[10] A. R. Cho, Y. J. Cha, H. R. Kim, E. K. Park, and E.-J. Cha, "Acquired amegakaryocytic thrombocytopenia after thymectomy in a case of pure red cell aplasia associated with thymoma," The Korean Journal of Laboratory Medicine, vol. 30, no. 3, pp. 244-248, 2010.

[11] C. M. Gay, W. N. William Jr., S. A. Wang, and T. H. Oo, "Thymoma complicated by acquired amegakaryocytic thrombocytopenia and pure red cell aplasia," Journal of the National Comprehensive Cancer Network, vol. 12, no. 11, pp. 1505-1509, 2014.

[12] A. Fujiwara, M. Inoue, H. Kusumoto, Y. Shintani, T. Maeda, and M. Okumura, "Myasthenic crisis caused by preoperative 
chemotherapy with steroid for advanced thymoma," The Annals of Thoracic Surgery, vol. 99, no. 1, pp. e11-e13, 2015.

[13] T. Onuki, Y. Kiyoki, S. Ueda, M. Yamaoka, S. Shimizu, and M. Inagaki, "Invasive thymoma with pure red cell aplasia and amegakaryocytic Thrombocytopenia," Hematology Reports, vol. 8, no. 4, p. 6680, 2016.

[14] B. H. G. Rogers, J. R. Manaligod, and W. V. Blazer, "Thymoma associated with pancytopenia and hypogammaglobulinemia," The American Journal of Medicine, vol. 44, no. 1, pp. 154-164, 1968.

[15] S. Burrows and R. Carroll, "Thymoma associated with pancytopenia," Archives of pathology, vol. 92, no. 6, pp. 465-468, 1971.

[16] M. A. Chapin, "Benign thymoma, refractory anemia and hypogammaglobulinemia-case report," The Journal of the Maine Medical Association, vol. 56, pp. 83-87, 1965.

[17] D. Korn, A. Gelderman, G. Cage, D. Nathanson, and A. J. L. Strauss, "Immune deficiencies, aplastic anemia and abnormalities of lymphoid tissue in thymoma," New England Journal of Medicine, vol. 276, no. 24, pp. 1333-1339, 1967.

[18] M. A. Mir, C. G. Geary, and I. W. Delamore, "Hypoimmunoglobulinaemia and aplastic anaemia," Scandinavian Journal of Haematology, vol. 19, no. 3, pp. 225-229, 1977.

[19] H. A. Kristiansen, S. Fløisand, Y. Fløisand, and D. Heldal, "Eltrombopag in good's syndrome," Case Reports in Hematology, vol. 2014, Article ID 172139, 3 pages, 2014.

[20] V. Trisal, A. Nademanee, S. K. Lau, and F. W. Grannis Jr., "Thymoma-associated severe aplastic anemia treated with surgical resection followed by allogeneic stem-cell transplantation," Journal of Clinical Oncology, vol. 25, no. 22, pp. 3374-3375, 2007.

[21] A. Numata, K. Yasuda, T. Fukuda et al., "Non-myeloablative allogeneic haemopoietic stem-cell transplantation for treatment of metastatic invasive thymoma," The Lancet Oncology, vol. 6, no. 8, pp. 626-628, 2005.

[22] C. Lu, G.-S. He, S. Jin et al., "Haploidentical allogeneic hematopoietic stem cell transplantation for thymoma-associated severe aplastic anemia: a case report," Chinese Medical Sciences Journal, vol. 28, no. 3, pp. 189-191, 2013.

[23] A. Gaglia, A. Bobota, E. Pectasides, C. Kosmas, G. Papaxoinis, and D. Pectasides, "Successful treatment with cyclosporine of thymoma-related aplastic anemia," Anticancer Research, vol. 27, no. 4C, pp. 3025-3028, 2007.

[24] M. J. Olnes, P. Scheinberg, K. R. Calvo et al., "Eltrombopag and improved hematopoiesis in refractory aplastic anemia," New England Journal of Medicine, vol. 367, no. 1, pp. 11-19, 2012.

[25] C. Rivoisy, B. Besse, N. Girard et al., "Thymic epithelial tumor-associated cytopenia: a 10-year observational study in France," Journal of Thoracic Oncology, vol. 11, no. 3, pp. 391-399, 2016.

[26] J. P. Novotný, B. Köhler, R. Max, and G. Egerer, “Acquired amegakaryocytic thrombocytopenic purpura progressing into aplastic anemia," Prague Medical Report, vol. 118, no. 4, pp. 147-155, 2017. 


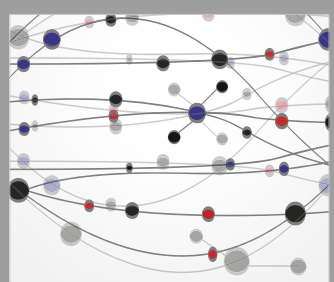

The Scientific World Journal
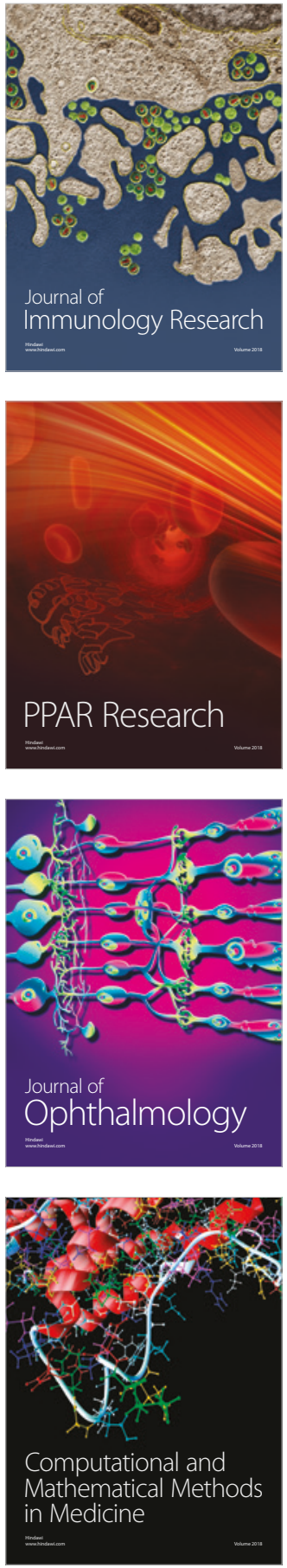

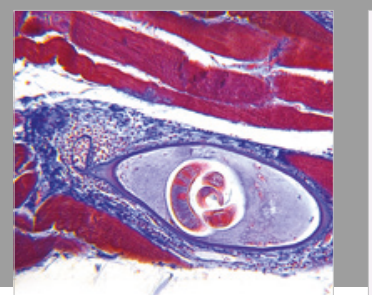

Gastroenterology Research and Practice

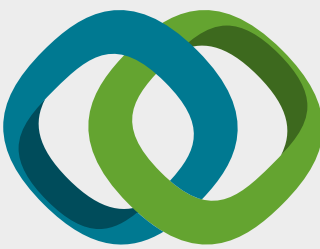

\section{Hindawi}

Submit your manuscripts at

www.hindawi.com
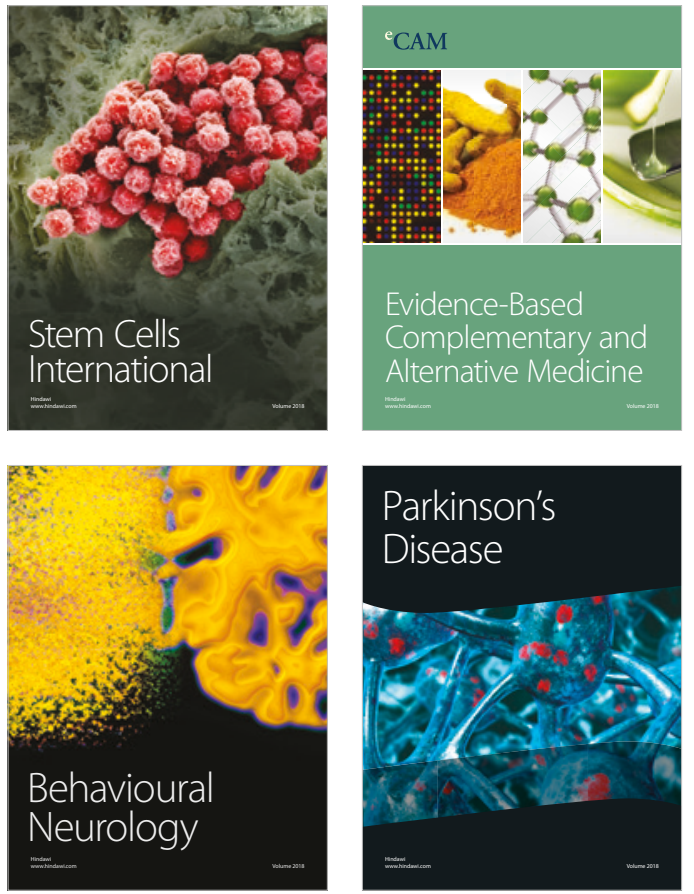

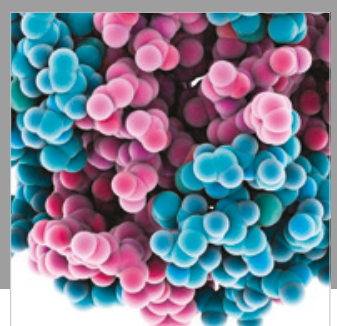

ournal of

Diabetes Research

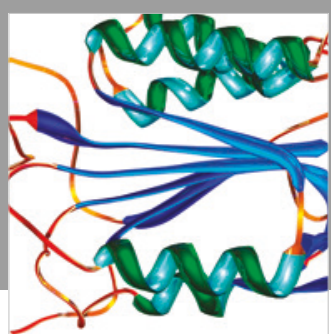

Disease Markers
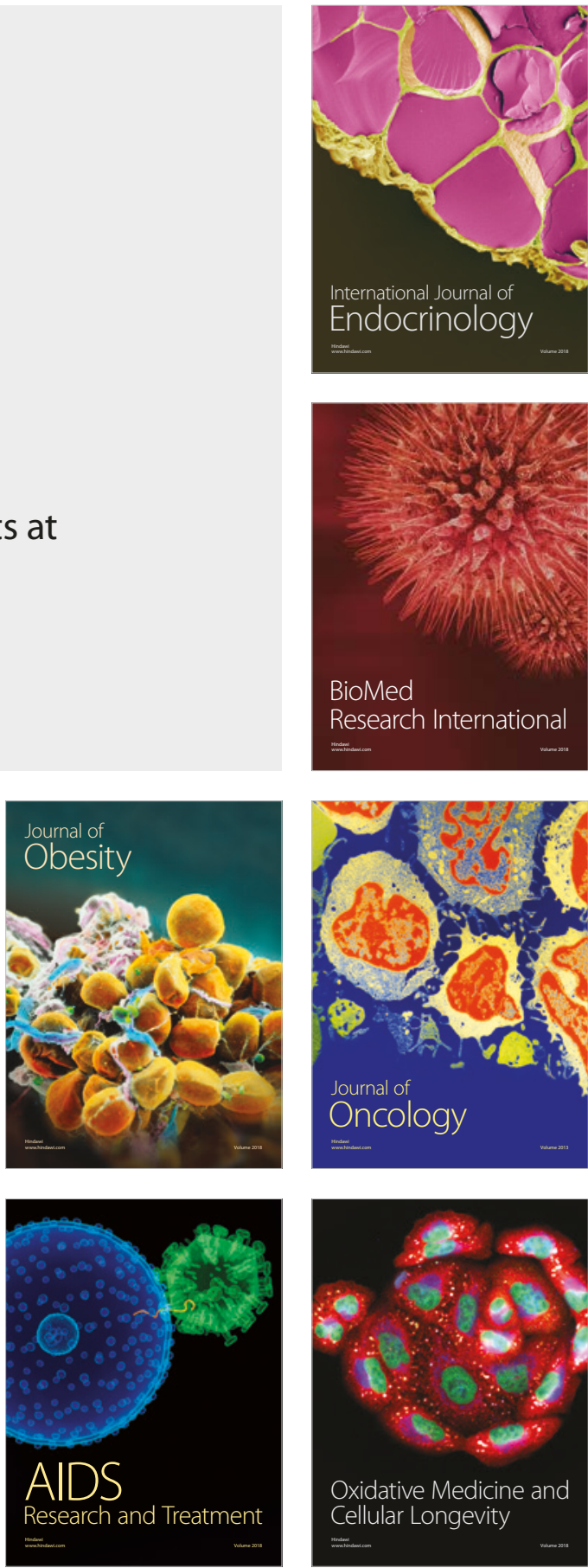